\title{
Les couches de forme traitées dans les infrastructures ferroviaires
}

\section{A. ROBINET}

SNCF

Direction de l'ingénierie

Département des ouvrages d'art 6 , avenue

François-Mitterrand

93574 La Plaine Saint-Denis alain.robinet@sncf.fr
La SNCF et RFF ont mené un programme de recherche afin de valider la faisabilité et le dimensionnement des couches de forme réalisées en matériaux traités afin d'optimiser la réutilisation des matériaux du site. L'objectif de la recherche est de définir les spécifications à imposer à la couche de forme traitées afin de prendre en compte les particularités de l'environnement ferroviaire telles que l'environnement hydrique, les sollicitations vibratoires et l'impact de l'infrastructure sur le vieillissement de la voie. Le programme comprend quatre parties : (i) analyse du comportement d'une zone expérimentale de $17 \mathrm{~km}$ réalisée avec une couche de forme en sable traitée en 1990 ; (ii) prélèvement et analyses d'échantillons sur cette zone ; (iii) fabrication et sollicitations de planches de test en grandeur réelle au CER de Rouen; (iv) modélisation afin de vérifier le comportement à la fatigue d'une structure ferroviaire intégrant une couche de forme traitée. Cet article présente les travaux réalisés dans le cadre de ce programme.

Mots-clés : infrastructure ferroviaire, couche de forme, sol traité, essai en grandeur réelle, fatigue, modélisation.

\section{Treated capping layers in railway infrastructure}

The SNCF (French railways operator) and RFF (French railways owner) have launched a research program in order to validate the feasibility and the design of capping layers made of treated soil with the aim of allowing the use of the soil from the site of the railway infrastructure construction. The objective of the research was to define the complementary specifications to the capping layer of treated soil when considering the particularities associated with railways lines, such as the humid environment, the vibratory stresses and the impact on the ageing of the track. The program is comprised of four parts: (i) analysis of the behaviour of a maintenance zone of $17 \mathrm{~km}$ on the high speed line Atlantique (LGV A) where an experimental section of capping layer made of treated sand was implemented in 1990; (ii) sampling on the LGV A and analyzing the behaviour of the treated soil; (iii) performing full scale tests in the CER (Road Studies Centre) of Rouen allowing a comparison to be made between the traditional granular behaviour of structure and structures treated with road hydraulic binders;

(iv) modelling the fatigue of the railway structures integrating the capping layers made of treated soil. This paper presents the investigations performed within this program.

Key words: railway infrastructure, capping layer, treated soil, full scale test, fatigue, modelling. 


\section{Introduction}

Le catalogue des structures d'assise des voies nouvelles en vigueur à la SNCF prévoit la mise en place d'une couche de forme de 35 à $50 \mathrm{~cm}$ en fonction du sol support. La couche de forme est réalisée en matériaux granulaires correspondant à des spécifications relativement précises rendant très difficile l'utilisation des matériaux du site. Bien que les couches de forme en matériaux traités à la chaux et aux liants hydrauliques soient aujourd'hui couramment utilisées dans le domaine routier et que leur conception et mise en œuvre soient normalisées, il n'en est pas de même dans le domaine ferroviaire où l'application de cette technique n'est prévue dans aucun référentiel, aussi bien en France que dans les réseaux étrangers membres de l'UIC (Union internationale des chemins de fer). La transposition de la technique des couches de forme traitées au milieu ferroviaire nécessite des investigations complémentaires permettant de s'assurer :

- du dimensionnement nécessaire pour assurer la pérennité du système sous sollicitations ferroviaires et dans un milieu hydrique plus défavorable;

- de la (c transparence » sur la maintenance ultérieure de l'ouvrage.

Cette étude pourrait amener à des aménagements dans l'application aux infrastructures ferroviaires du document de référence de la technique routière, le guide des traitements de sols (le GTS).

Pour répondre à ces questions, le RFF et la Direction de l'ingénierie de la SNCF ont entrepris une étude articulée en trois volets :

- analyse d'une zone de la ligne à grande vitesse (LGV) Atlantique où ont été mises en œuvre expérimentalement des couches de forme traitées

- suivi de planches expérimentales en laboratoire;

- modélisation.

On présente ici successivement les trois volets de la recherche et des conclusions sur la transposition de la technique routière au monde ferroviaire. L'objectif final est de permettre de respecter les contraintes économiques et celles liées au développement durable en optimisant la réutilisation des matériaux du site.

\section{2}

\section{Analyse de la zone expérimentale de la LGV Atlantique}

La ligne à grande vitesse ( Atlantique ) (LN2) a été inaugurée en 1990. Sur la branche ( Bretagne ) (tronçon entre Courtalain et Le Mans), plusieurs zones ont été réalisées à titre expérimental avec des couches de forme traitées en sable ciment, correspondant à un total de $17 \mathrm{~km}$ de ligne.

En première approche, le REX (retour d'expérience) a consisté à comparer la qualité de nivellement de la voie et l'effort de maintenance entre les zones comportant des couches de forme traitées et les zones comportant les couches de forme granulaires. Par la suite, on a prélevé puis testé en laboratoire 18 échantillons carottés dans la couche de forme traitée pour étudier ses caractéristiques résiduelles après 17 années d'exploitation.

\section{Comparaison entre les zones traitées et les zones classiques}

L'opération de base de maintenance de la géométrie des voies ferrées est le bourrage, opération qui consiste à replacer la voie dans la position souhaitée et à vibrer le ballast. Il s'agit donc ici de comparer la fréquence des opérations de bourrage entre les zones réalisées avec des structures classiques et celles réalisées avec des couches de forme traitées.

Sur la figure 1, les zones traitées (T) et non traitées (NT) sont indiquées. Les zones sont présentées en PK croissant du PK 132 au PK 180. Ce graphique montre une certaine hétérogénéité en fonction de la localisation de la zone, le traitement n'ayant pour sa part aucune influence identifiée.

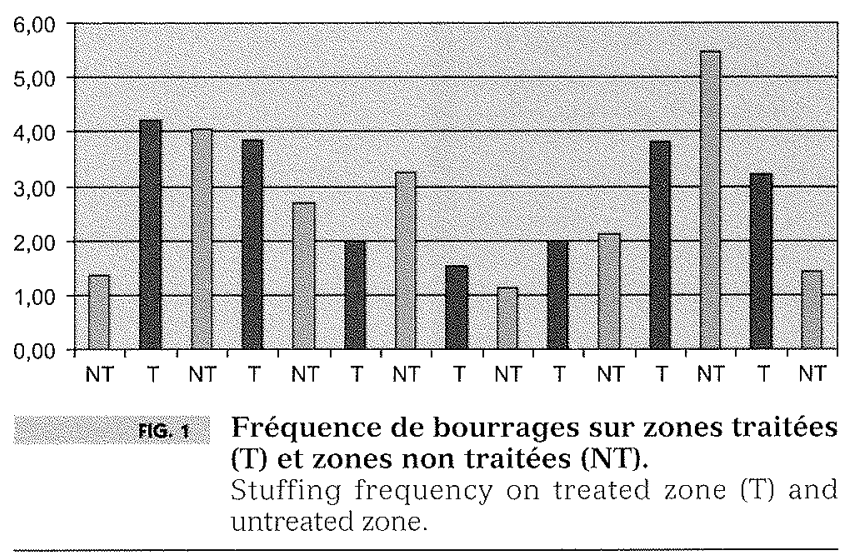

\section{Prélèvements des matériaux}

On a prélevé des carottes sur 18 points représentant l'ensemble des zones des échantillons de la couche de forme traitée. Ces échantillons ont fait l'objet de diverses caractérisations :

- examen visuel ;

- mesure de masse volumique;

- détermination de la résistance mécanique ;

- détermination de la perméabilité ;

- étude du ciment résiduel.

L'examen visuel montre un bon aspect des carottes. Les quelques fissures détectées sont horizontales et présentent un aspect très « frais ». Elles peuvent être imputées au mode de prélèvement. Une seule carotte présente un aspect fragmenté ; il s'agit d’un prélèvement réalisé en piste, le gel est sans doute à l’origine de la fragmentation comme on, le verra plus bas. L'épaisseur de couche traitée est d'en moyenne $42 \mathrm{~cm}$.

La mesure de la masse volumique montre une bonne homogénéité sur l'épaisseur de la couche de matériaux traités. Le tableau I montre la valeur moyenne et l'écart-type par carotte.

Les caractéristiques mécaniques mesurées sont dispersées mais proches des valeurs habituelles:

- Rtb (résistance à la traction avec l'essai brésilien) de 0,44 à 1,17 MPa avec une moyenne de $0,80 \mathrm{MPa}$; 
Et (module de traction) de 5349 à $13183 \mathrm{MPa}$ avec une moyenne de $9378 \mathrm{MPa}$;

- Ec (module de compression) de 5136 à $6617 \mathrm{MPa}$ avec une moyenne de $5660 \mathrm{MPa}$.

La figure 2 montre qu'il n'apparaît pas de corrélation entre les caractéristiques mécaniques et les masses volumiques mesurées. Les caractéristiques mécaniques du matériau traité le situent entre les classes mécaniques 2 et 3 selon le Guide de traitement des sols du LCPC/SETRA (Fig. 3). Les mesures de perméabilité se situent entre $7 \cdot 10^{-7}$ et $3.10^{-6} \mathrm{~m} / \mathrm{s}$, ce qui amène à qualifier le matériau de ( faiblement perméable ). La quantité et la composition chimique du liant analysées restent identiques à celles mises en ouvre.

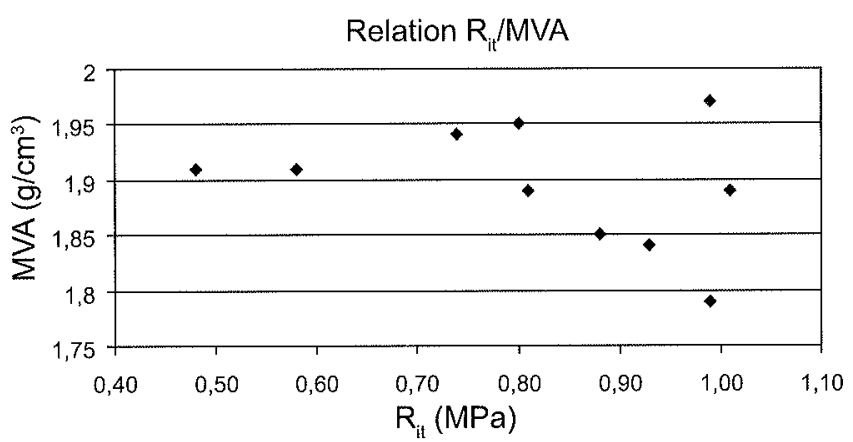

Ho. 2 Relation résistance en traction/masse volumique.

Relationship between extension shear strength and unit mass.

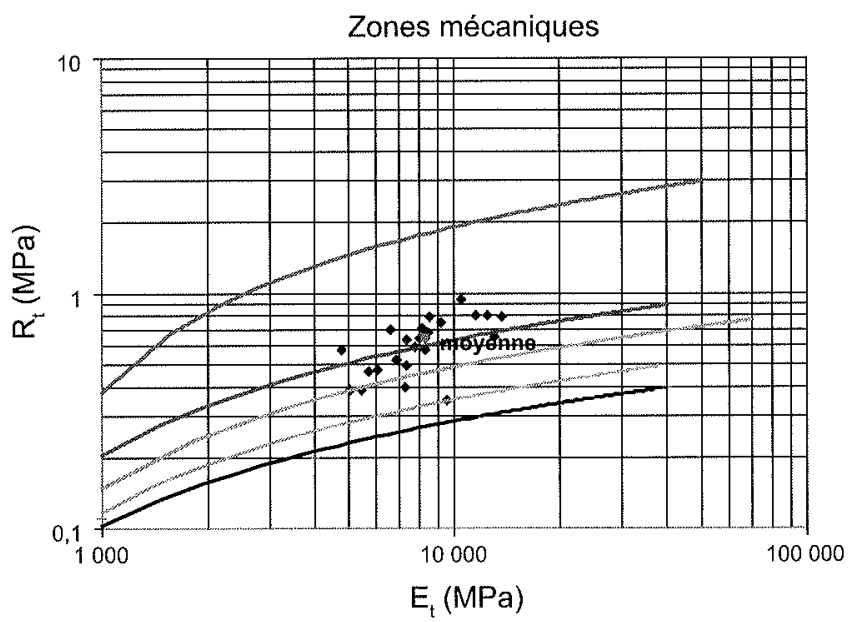

16. 3 Zone mécanique de la couche de forme. Mechanical zone of the capping layer.

\section{Analyse des épisodes de gel}

Considérons les couches amenant une protection au-dessus de la couche de forme. En prenant une épaisseur forfaitaire de $15 \mathrm{~cm}$ pour la traverse, une épaisseur de ballast de $35 \mathrm{~cm}$ et $20 \mathrm{~cm}$ de sous-couche, l'épaisseur totale est de $70 \mathrm{~cm}$. Ces couches, en acceptant l'approximation de les assimiler à un matériau granulaire homogène amène, en suivant le catalogue des structures de chaussée de 98, une protection contre la pénétration au gel de $100^{\circ} \mathrm{C}^{*}$ jours.

L'exploitation des données climaticues de la station Météo France de Cormes montre que depuis la création de la ligne, il y a eu deux épisodes de gel amenant à un indice supérieur :

-1991 : indice $=101^{\circ} \mathrm{C}$ jour ;

-1997 : indice $=140^{\circ} \mathrm{C}^{*}$ jour.

Dans ces conditions, la couche de forme a subi l'agression du gel sur une épaisseur d'environ $15 \mathrm{~cm}$ depuis la mise en service de la ligne.

Le Guide de traitement des sols admet le critère de non-gélivité des matériaux pour une $R_{t}>0,25 \mathrm{MPa}$. Cette condition est vérifiée pour la quasi-totalité des points mesurés qui présentent une $R_{t}$ issue de la $R_{i t}$ comprise entre 0,35 et 0,94 . La seule exception provient du point de sondage 14 qui présente une $R_{i t}$ de $0,28 \mathrm{MPa}$ soit une $\mathrm{R}_{\mathrm{t}}$ de $0,22 \mathrm{MPa}$.

Rappelons que ce point de sondage est situé en piste et que l'examen visuel avait révélé une dégradation importante du prélèvement. Ce qui vient d'être dit permet de justifier l'hypothèse qui avait été prise de dégradation du matériau par le gel, le matériau étant localement non protégé (piste) et gélif $\left(R_{t}<0,25 \mathrm{MPa}\right)$. Cela montre également que le critère proposé par le GTS est justifié, pour ce matériau tout au moins.

\section{Planches d'essai au CER de Rouen}

Trois planches d'essai conformes à la figure 4 ont été réalisées en laboratoire. Le sol support était constitué d'un sable limoneux. Le matériau traité était un sable. Une sous-couche de $17 \mathrm{~cm}$ de GNT 0/31,5 était mise en œuvre entre le matériau traité et le ballast. La structure classique était constituée d'une sous-couche de GNT 0/31,5 sur une épaisseur de $55 \mathrm{~cm}$. Les planches ont été sollicitées selon le chargement cyclique décrit sur la figure 5.

L'objectif des essais était l'étude du comportement du matériau traité dans le milieu ferroviaire

- sous sollicitations cycliques;

- en présence d'eau.

Les résultats montrent la pérennité de la structure. Les déformations sont beaucoup plus faibles (rapport de 3 à 1) et les accélérations sont largement diminuées avec les structures traitées (Figs. 6 et 7).

Alors que l'on craignait une dégradation prématurée du ballast, les planches d'essai ont montré à terme une modification granulaire de la sous-couche (Fig. 8) avec une production de fines plus importantes dans le cas des structures traitées. L'état hydrique des matériaux pendant les phases de sollicitations n'est pas étranger à la dégradation prématurée des matériaux de sous-couche.

\section{Modélisation}

L'introduction de matériaux traités dans la structure ferroviaire nécessite un dimensionnement à la fatigue permettant de justifier la pérennité de la couche traitée. La vérification a été réalisée conformément à la méthode française de dimensionnement des chaussées. Le calcul des contraintes et déformations dans 


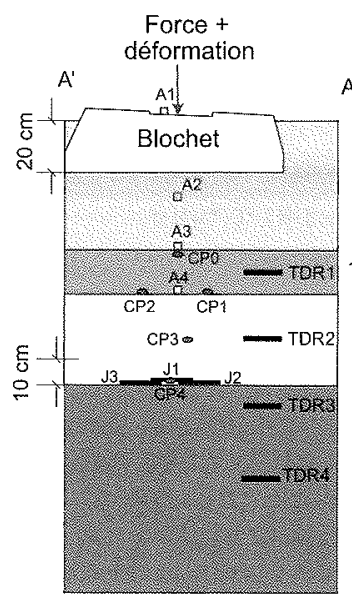

Structures traitées à $4 \%$

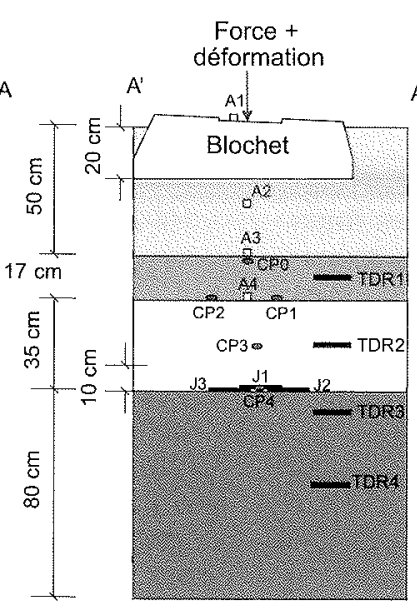

Structures traitées à $6 \%$

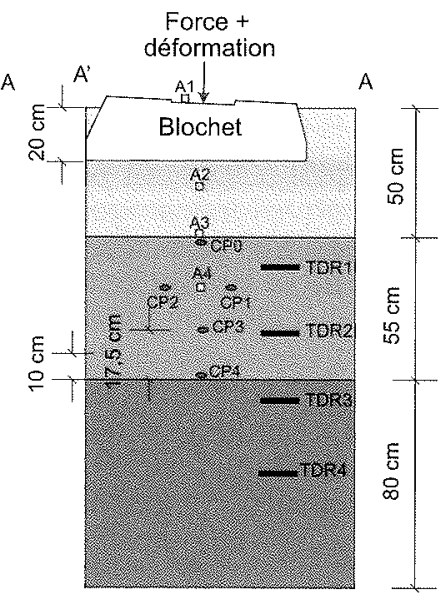

Structure classique

\begin{tabular}{|c|c|c|c|}
\hline \multicolumn{4}{|c|}{ LÉGENDE } \\
\hline a & A & : & Accéléromètre \\
\hline$\infty$ & $\mathrm{CP}$ & : & Capteur de pression \\
\hline & TDR & : & Teneur en eau \\
\hline & & & \\
\hline
\end{tabular}

F6. 4 Planches d'essai au laboratoire du CER de Rouen.

Test boards in the laboratory of CER of Rouen.

\section{Force à appliquer}

$(\mathrm{KN})$

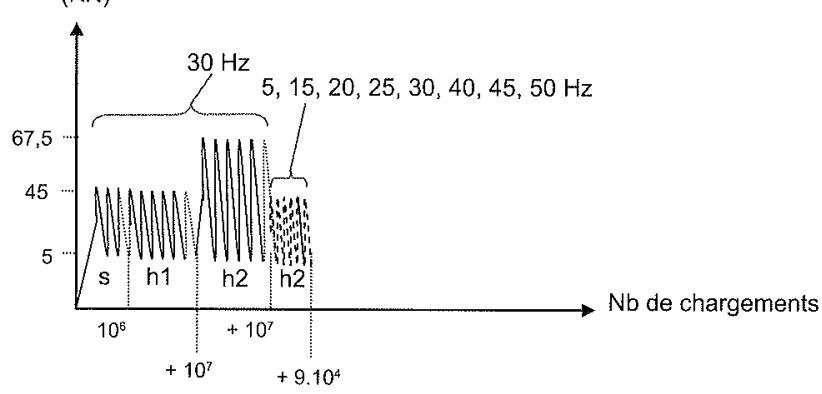

s: $\sec$

$\mathrm{h} 1$ : humide aggravation de l'état $)=40 \mathrm{~mm} / \mathrm{j} \mathrm{r}^{*} 10 \mathrm{j}=1$ pluviométrie sur 6

mois de l'année $\rightarrow$ quasi-saturation

$\mathrm{h} 2$ : humide (maintien de l'état) $=10 \mathrm{~mm} / \mathrm{jr} * 10 \mathrm{j}=$ maintien proche de la saturation

I6. 5 Chargement cyclique des planches d'essai. Cyclic loading on test boards.
Moyenne des accélérations en fonction de la profondeur Phase humide $\mathrm{H} 1$

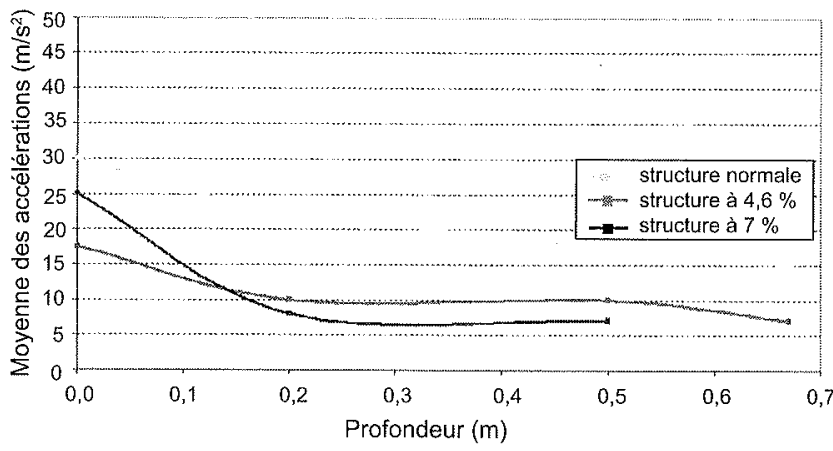

月6.7 Mesure des accélérations. Acceleration measurement.
Phases $\mathrm{H}_{1}$ ef $\mathrm{H}_{2}$

Estructure normale - structure $4,6 \%=$ sfructure $7 \%$

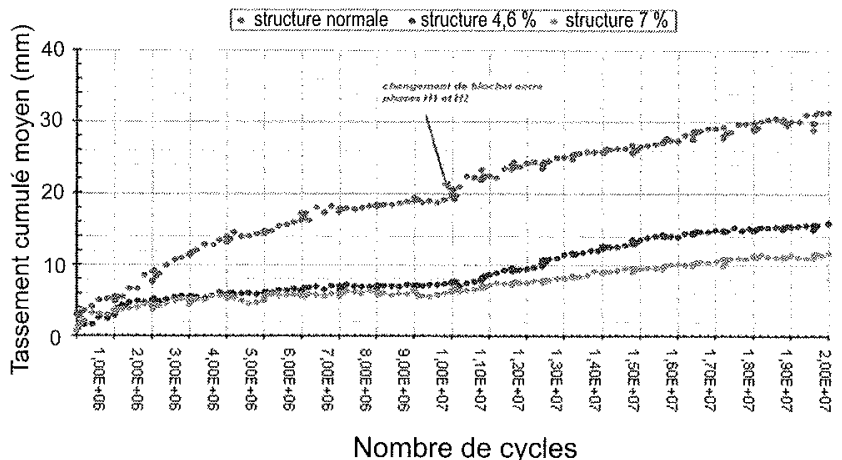

2.1. 16 . 6 . Tassement cumulé moyen. Mean cumulated settlement.

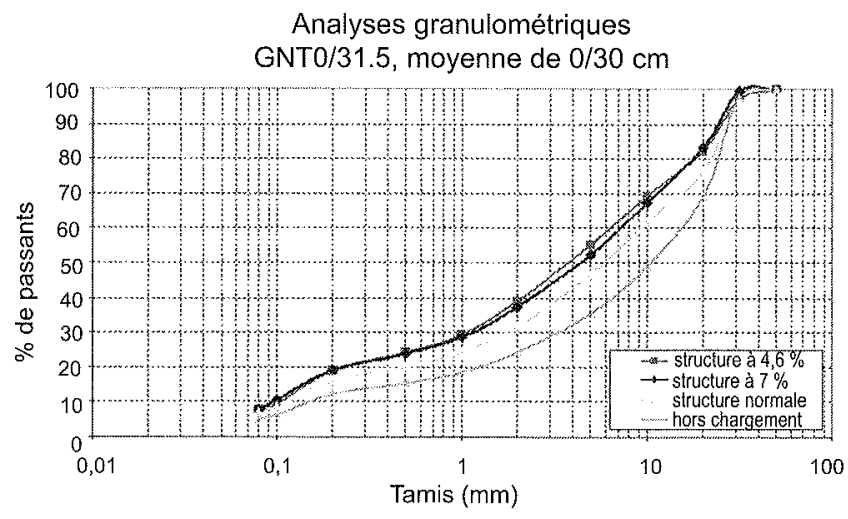

ra. 8 Évolution granulométrique de la souscouche.

Evolution of the grain size distribution of the capping layer. 
les couches constitutives a été avec le logiciel César. Les contraintes et déformations admissibles ont été calculées selon la méthode LCPC en tenant compte du projet de guide technique Application à la réalisation des assises de chaussée des matériaux traités du CFTR pour l'estimation du $\sigma 6$ en particulier. Le calcul a été fait pour divers trafics TGV sur des durées de vie de 60 et 100 ans.

5

\section{Conclusion}

Les différentes études ont montré la faisabilité technique de la solution couche de forme traitée dans les structures d'assise ferroviaire. Le dimensionnement sera à vérifier au cas par cas selon les règles de calcul du dimensionnement à la fatigue en fonction du trafic attendu, du matériau à mettre en place et de la qualité de sol support. Un soin tout particulier devra être apporté pour assurer l'homogénéité du produit comprenant :

- homogénéité du gisement ;

- homogénéité du traitement ;

- homogénéité du comportement dans le temps.

Cette étude a permis de modifier le référentiel des infrastructures des lignes à grande vitesse pour y intégrer cette nouvelle possibilité. 